\title{
Pulmonary actinomycosis: cytomorphological features
}

\author{
Rafael Martínez-Girón', Liron Pantanowitz ${ }^{2}$ \\ ${ }^{1}$ INCLÍNICA Foundation for Clinical, Pneumological and Carcinogenic Research, Oviedo, Spain; ${ }^{2}$ Department of \\ Pathology and Clinical Labs, University of Michigan, Ann Arbor, MI, USA
}

\begin{abstract}
Pulmonary actinomycosis is an uncommon infectious disease. Although the gold standard for diagnosis is histological examination with bacterial culture of lung tissue, cytology samples offer a fast and low-cost alternate diagnostic procedure. The cytology literature on this topic is limited to mostly case reports. Therefore, the aim of this study was to review cytological material in a series of patients with a diagnosis of pulmonary actinomycosis to characterize the main cytomorphological findings. Different cytological respiratory samples including sputum smears, bronchoalveolar lavages (BALs), transthoracic or endobronchial fine needle aspiration cytol-
\end{abstract}

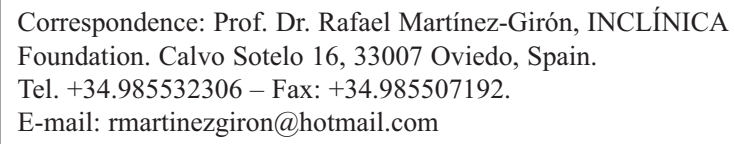

E-mail: rmartinezgiron@hotmail.com

Key words: Pulmonary actinomycosis; cytology; sputum; fine needle aspiration; cell blocks

Contributions: Both authors have contributed equally. All the authors have read and approved the final version of the manuscript and agreed to be accountable for all aspects of the work.

Conflict of interest: The authors declare no potential conflict of interest with this manuscript.

Availability of data and material: The datasets used and/or analysed during the current study are available from the corresponding author on reasonable request.

Ethics approval: This study was conducted in accordance with the Code of Ethics of the World Medical Association (Declaration of Helsinki).

Informed consent: Informed consent was not requested from all participants included in this study due to retrospective study design.

Received for publication: 15 October 2020.

Accepted for publication: 22 September 2021.

${ }^{\circ}$ Copyright: the Author(s), 2021

Licensee PAGEPress, Italy

Monaldi Archives for Chest Disease 2022; 92:1641

doi: 10.4081/monaldi.2021.1641

This article is distributed under the terms of the Creative Commons Attribution Noncommercial License (by-nc 4.0) which permits any noncommercial use, distribution, and reproduction in any medium, provided the original author(s) and source are credited. ogy (FNAC) and cell block preparations were used for retrospective examination. For all cases patient age, gender, symptoms, and radiological chest findings were recorded. A total of 26 cytological respiratory samples (14 sputum smears, 9 FNAC, two BALs) including direct smears and 6 cell blocks from 9 patients were examined. In sputum smears the most remarkable findings were the presence of dark cotton ball masses with projections like spider legs and/or mouse tails ( $75 \%$ of the samples). Sulfur granules were observed in $4(40 \%)$ of the sputum smears and within FNAC cases. Various respiratory cytology samples including sputum smears, FNAC and BALs can reveal cytomorphological findings diagnostic of pulmonary actinomycosis. Characteristic cytological findings compatible with a diagnosis of this infection include cotton ball masses and less frequently sulfur granules.

\section{Introduction}

Pulmonary actinomycosis is an uncommon infectious disease caused by Actinomyces spp., a genus of Gram-positive bacilli (1 $\mu \mathrm{m}$ in diameter) characterized by a growing filamentous pattern of these Actinobacteria with or without branching. Although currently 47 species of these bacteria have been identified, only six of them are incorporated in the genus Actinomyces, including $A$. israelii, A naeslundii, A. odontolyticus, A. viscosus, A. meyeri and A. pyogenes. A. israelli is g the most prevalent species to be isolated in human infections and has been found in most clinical cases of actinomycosis [1]. Some species are strict anaerobes and others are microaerophilic, all of which are slow growing.

This bacterial genus is part of the microbiota of the digestive tract, ranging from the oropharynx to the colon and is of low pathogenic potential [2]. The genitourinary tract is also a site of colonization. They may cause infections when they cross epithelial barriers under certain conditions that produce low oxygen tension, mainly in cases of tissue necrosis [3]. Thus, these infections usually occur endogenously such as after trauma, surgery and, quite often due to the presence of foreign bodies [4-8]. In older people with poor buccal hygiene and/or dental disease, whether or not accompanied by alcoholism, aspiration of oropharyngeal secretions containing these bacilli is one of the main forms of disease acquisition [9], although hematogenous spread from a distant infectious focus would be another infrequent source of infection $[10,11]$.

Pulmonary actinomycosis constitutes approximately $15-20 \%$ of all cases of this infectious disease [12]. Pulmonary actinomycosis may manifest itself in various ways, mainly affecting the lung parenchyma with extension to the chest wall $[13,14]$. Many cases can be clinically mistaken for other diseases such as fungal infection (e.g., Aspergillosis), tuberculosis, and lung cancer [15-19]. Pulmonary actinomycosis may occur at any age, but it is more frequent in people aged between 30 and 65 years old. It is also more 
frequent in men than in women. The clinical manifestations of pulmonary actinomycosis include fever, cough with phlegm (sputum), chest pain, dyspnea and hemoptysis [20]. Radiological findings may be non-specific, including the presence of masses, nodules, patchy infiltrates, segmental air-space consolidation, and cavitation $[21,22]$.

The gold standard for diagnosing pulmonary actinomycosis is histological examination of tissue (e.g., lung biopsy) revealing the typical oval shaped "sulfur granules" along with bacterial culture [23]. However, pulmonary cytology samples provide an alternate faster and low-cost diagnostic procedure without the need for invasive lung procedures allowing afflicted patients to receive early treatment of this infectious disease [24]. However, the cytology literature on this topic is limited to mostly case reports. Therefore, the aim of this study was to review cytological material in a series of patients with a diagnosis of pulmonary actinomycosis to characterize the main cytomorphological findings.

\section{Materials and Methods}

In this retrospective study, different cytology respiratory samples from patients with a confirmed diagnosis of pulmonary actinomycosis were used for examination under a light microscope. Cytology samples included sputum smears, bronchoalveolar lavages (BALs), and transthoracic or endobronchial fine needle aspiration cytology (FNAC). Available cell blocks were also studied. Depending on the type of sample, different staining methods were employed such as Papanicolaou, Giemsa, Hematoxylin and Eosin (H\&E), Periodic Acid Schiff (PAS), Grocott-Gomori and Gram stains.

In addition, the clinical records of included patients were searched to record age, gender, dental hygiene, alcoholism, symptoms, and radiological chest findings. The data presented in this article were collected as part of the routine work in a cytology lab- oratory. The study was undertaken in full compliance with the principles laid out in the Declaration of Helsinki.

\section{Results}

\section{Clinical findings}

The mean patient age was 68.6 years (range, 55 to 79 years) and all patients were males. In five patients $(55.5 \%)$ poor dental hygiene was documented, two patients $(22.2 \%)$ were diabetics and one $(11.1 \%)$ was an alcoholic. Among their clinical symptoms, cough with phlegm and fever were the most frequent (100\% of the cases), followed by hemoptysis $(66.6 \%)$, dyspnea and weigh loss (55.5\%), and finally chest pain (44.4\%). Unilateral cavitary lesions were the most frequent type of radiological lesion observed in $5 / 9$ $(55.5 \%)$ of the cases (Figure 1 A,B) in both thorax x-ray and computerized tomography (CT) studies. Other radiological findings included a unilateral mass-like shadow in 2/9 (22.22\%) of cases, unilateral consolidation in 1/9 (11.1\%) cases, and bilateral patchy infiltrates in in $1 / 9(11.1 \%)$ cases. All patients received antibiotic therapy for 2-6 weeks ( 7 of them with intravenous penicillin $\mathrm{G}$ and two with oral amoxicillin/clavulanic). Only two patients required surgical resection of the lesions. In all patients there was improvement of the symptoms and cure of the infection.

\section{Cytology findings}

A total of 31 cytology respiratory samples (14 sputum smears, 9 FNAC, two BALs), including 6 cases containing cell blocks, from 9 patients were examined. Of the sputum smears examined, 4 were considered unsatisfactory due to the existence of predominantly saliva with abundant pavimentous cells without the presence of alveolar macrophages. Hence, only 10 sputum smears were included in this study. In these sputum smears, the most remarkable findings were the presence of dark cotton ball-like masses with projections resembling spider legs and/or mouse tails
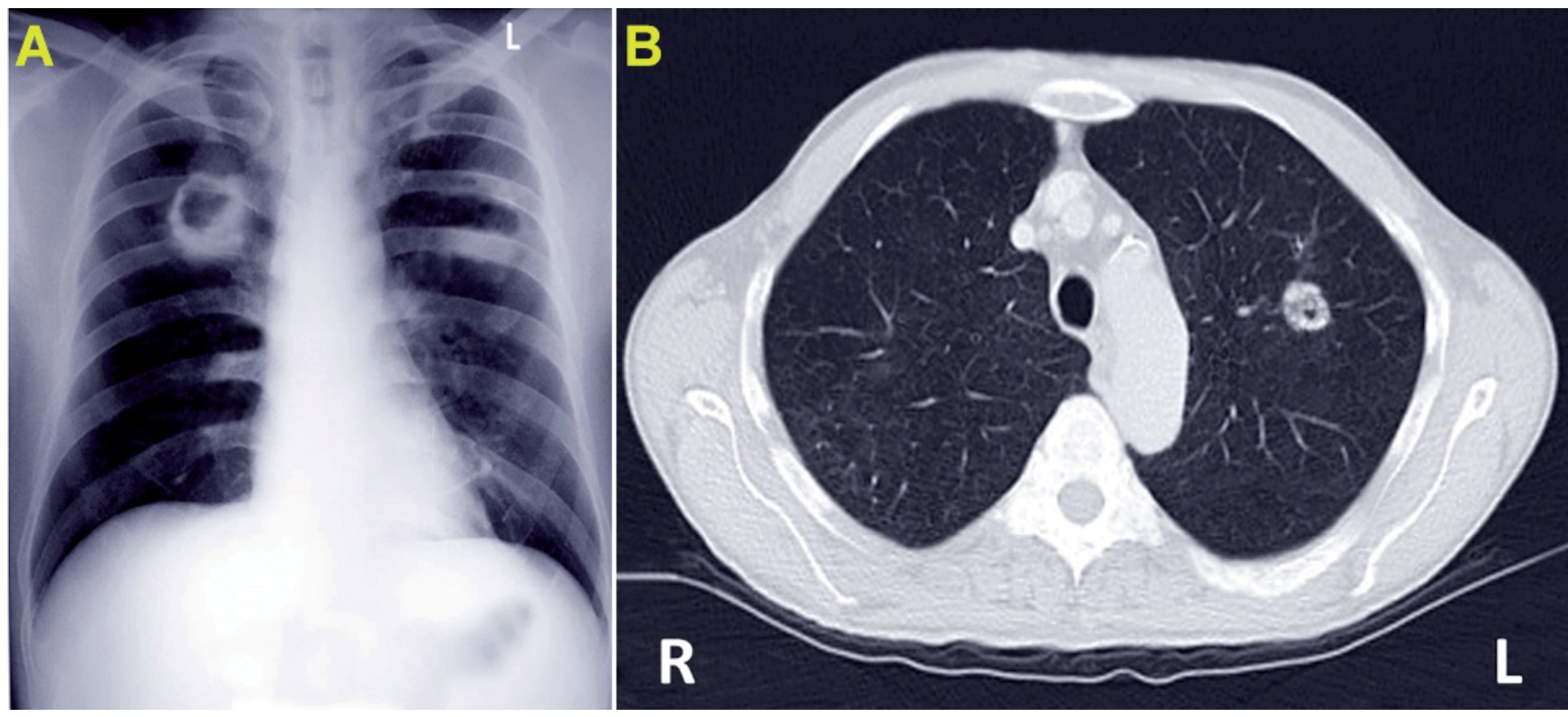

Figure 1. A) Chest $x$-ray showing a unilateral cavitary lesion in the right lung superior lobe. B) CT scan showing a unilaterally cavitary lesion in the left lung. 
(Figure $2 \mathrm{~A}, \mathrm{~B})$. This finding was observed in 7 of the 10 examined sputa $(75 \%)$. Less frequently, the presence of sulfur granules was observed (Figure 2C). This finding was observed in only 4 of the 10 examined sputum smears $(40 \%)$. In one of the two BALs elongated axis-like structures with numerous filiform projections was detected (Figure 2D). In FNAC samples the most characteristic finding was the presence of typical oval shaped sulfur granules, as masses of necrotic debris with radiating filaments and surrounded by inflammatory cells (Figure 3 A,B). Performing cell blocks, sulfur granules showed positivity with PAS, Giemsa and Grocott stains (Figure 4 B-D). This finding was observed in $100 \%$ of samples. The Splendore-Hoeppli phenomenon, characterized by intense eosinophilic material surrounding aggregated microorganisms, was seen in cell bock sections stained with H\&E (Figure 4A). In all respiratory samples microbiological cultures were carried out, showing positivity in all cases. Unfortunately, the details of the species isolated was unavailable. Other bacteria or fungi were not reported in these cultures.

\section{Conclusions}

Pulmonary actinomycosis is an uncommon disease, especially in developed countries today where social and hygiene habits have considerably improved. A study by Kim et al. accordingly included only 94 cases during the first decade of the $21^{\text {st }}$ century [25]. However, pulmonary actinomycosis remains a major cause of misinterpreted diagnoses related to lung infections [26]. For example, in a retrospective analysis of 145 cases by Zhang et al. only 5 patients had the correct initial diagnosis of primary pulmonary actinomycosis, and 60 patients were misdiagnosed with lung cancer [27]. In our study, 7 out of $11(64 \%)$ were initially clinically diagnosed as having lung cancer. In another retrospective study involving 26 patients with pulmonary actinomycosis, an initial misdiagnosis of lung cancer was made in $50 \%$ of cases, and pulmonary tuberculosis in another $26.9 \%$ [28]. While pulmonary actinomycosis can clinically mimic other pulmonary infections (e.g., nocardiosis, aspergillosis, tuberculosis) or even malignancy [24,29-33], it is important to be aware that co-infections with other microorganisms creating lung masses have also been described [34-37]. Aspiration of oropharyngeal secretions or the presence of a foreign body in the bronchial tree seems to be the main cause of pulmonary actinomycosis. Other forms of thoracic involvement include endobronchial disease, mediastinal infection, and pleural effusion [38-41]. The clinical (e.g., productive cough with fever) and radiological findings (e.g., cavitary lesion) in our group of patients were in line with other reported cases of pulmonary actinomycosis.

Compared to tissue biopsy and culture, cytology sample procurement from the respiratory tract provides a faster, cheaper, and less invasive mechanism to definitively establish a diagnosis of pulmonary actinomycosis. If actinomycetes are found only in sputum, usually without sulfur granules, they may merely reflect colonization [42], specially from the tonsillar crypts [43]. In such sputum specimens non-pathogenic actinomycetes tend to be juxtaposed with pyknotic squamous cells and sometimes even admixed with oropharyngeal Candida microorganisms. In our series, 8 of the 10 examined sputa $(80 \%)$ were positive for pulmonary actinomycosis and 4 of these samples (40\%) also had sulfur granules. Apart from sputum, other cytology samples such as FNAC and BAL have also been shown to be useful in the diagnosis of pulmonary actinomycosis [44-47]. Actinomycosis should always be considered when a FNAC of a lung mass contains an inflammatory exudate rich in polymorphonuclear neutrophils [48]. In such cases, cell block preparation along with special stains for microorganisms will improve diagnostic efficacy [49]. In our study, actinomycetes were observed with the aid of histochemical stains in $100 \%$ of the samples obtained by FNAC. Our study is limited by preforming a retrospective review and the low number of samples analyzed.

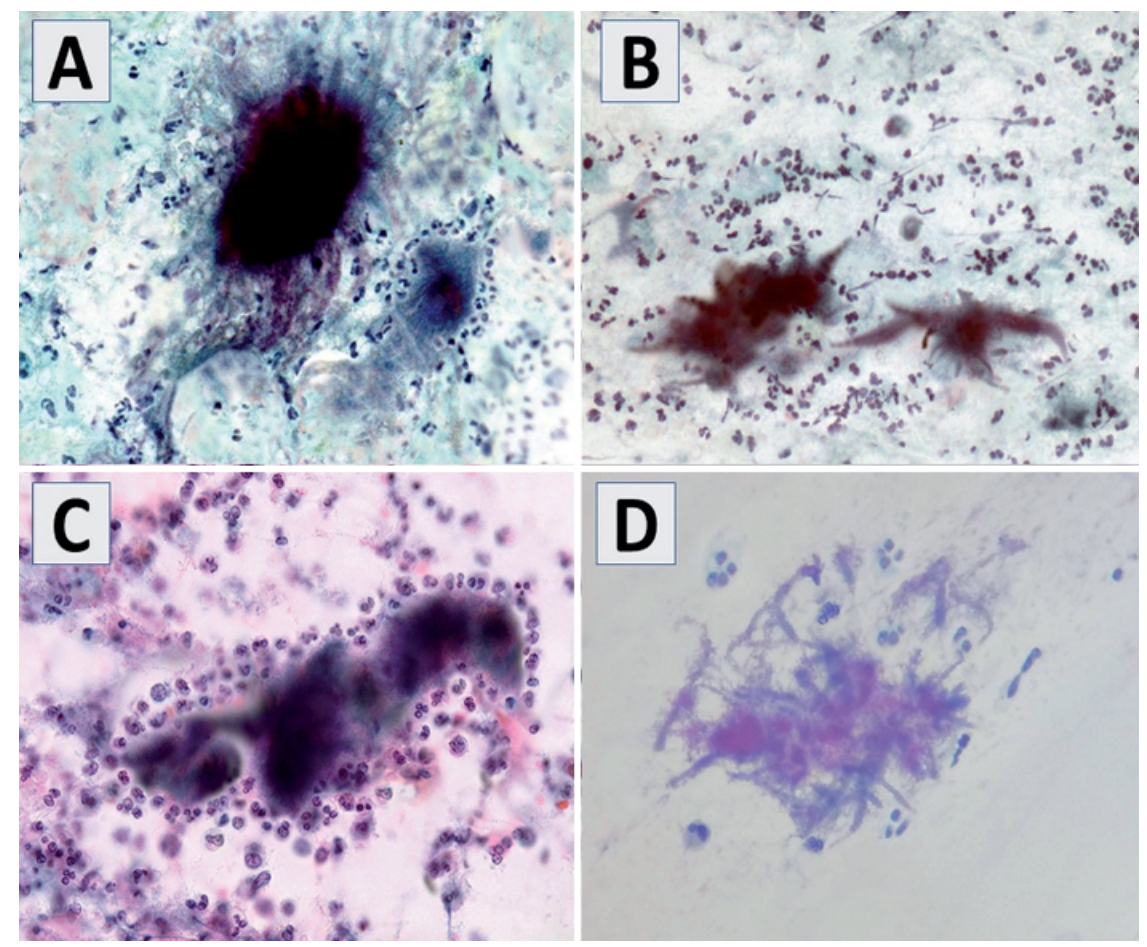

Figure 2. A) Sputum smear in pulmonary actinomycosis showing the presence of dark cotton ball-like masses with projections that resemble spider legs and/or mouse tails (Papanicolaou stain, x400). B) Sputum smear with dark cotton ball-like masses and mouse tail projections (Papanicolaou stain, x400). C) Sputum smear showing a typical sulfur granule (Papanicolaou stain, x400). D) BAL specimen with pulmonary actinomycosis showing elongated axis-like structures with numerous filiform projections

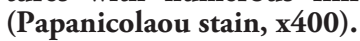


In conclusion, pulmonary actinomycosis is associated with characteristic cytological findings such as cotton ball-like masses and sulfur granules in respiratory specimens that can help make a timely and definitive diagnosis of this infection. The use of simple histochemical stains to identify actinomycetes embedded within these inflammatory structures can help confirm the diagnosis. This allows an early diagnosis and specific antibiotic therapy to be initiated, thereby avoiding unnecessary surgical intervention.

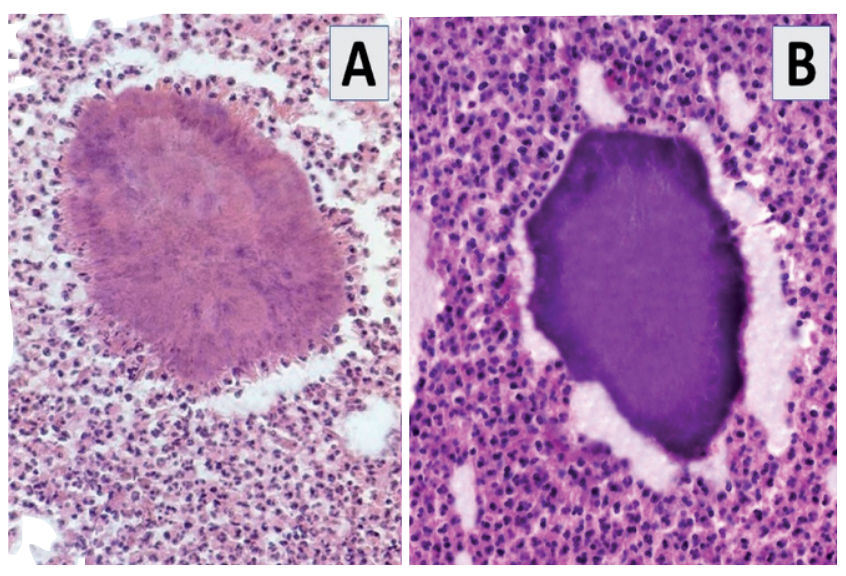

Figure 3. FNAC of pulmonary actinomycosis showing sulfur granules in cell block preparations surrounded by numerous acute inflammatory cells $(\mathrm{H} \& \mathrm{E}, \mathrm{x} 400)$.

\section{References}

1. Könönen E, Wade WG. Actinomyces and related organisms in human infections. Clin Microbiol Rev 2015;28:419-42.

2. Li J, Li Y, Zhou Y et al. Actinomyces and alimentary tract diseases: A review of its biological functions and pathology. Biomed Res Int 2018;2018:3820215.

3. Wong VK, Turmezei TD, Weston VC. Actinomycosis. BMJ 2011;343:d6099.

4. Godfrey AM, Diaz-Mendoza J, Ray C, Simoff MJ. Endobronchial actinomycosis after airway stenting. J Bronchology Interv Pulmonol 2012;19:315-8.

5. Julia G, Rodríguez de Castro F, Caminero J, et al. Endobronchial actinomycosis associated with a foreign body. Respiration 1991;58:229-30.

6. Murray MA, Rogan MP, Morgan RK, Linnane SJ. Bronchial dentures as a cause of airway actinomycosis. BMJ Case Rep 2014;2014: bcr2014204109.

7. Baek JH, Lee JH, Kim MS, Lee JC. Pulmonary actinomycosis associated with endobronchial vegetable foreign body. Korean J Thorac Cardiovasc Surg 2014;47:566-8.

8. Sobajima T, Asano F, Tsuzuku A, et al. A case of pulmonary actinomycosis associated with aspiration of cedar leaves. J Bronchology Interv Pulmonol 2015;22:259-62.

9. Mabeza GF, Macfarlane J. Pulmonary actinomycosis. Eur Respir J 2003;21:545-51.

10. Colmegna I, Rodriguez-Barradas $M$, Rauch $R$, et al. Disseminated Actinomyces meyeri infection resembling lung cancer with brain metastases. Am J Med Sci 2003; 326:152-5.

11. Lawson E. Systemic actinomycosis mimicking pelvic malignancy with pulmonary metastases. Can Respir J 2005;12:153-4.

12. Wong VK, Turmezei TD, Weston VC. Actinomycosis. BMJ 2011;343:d6099.

13. Farrokh D, Rezaitalab F, Bakhshoudeh B. Pulmonary actino-
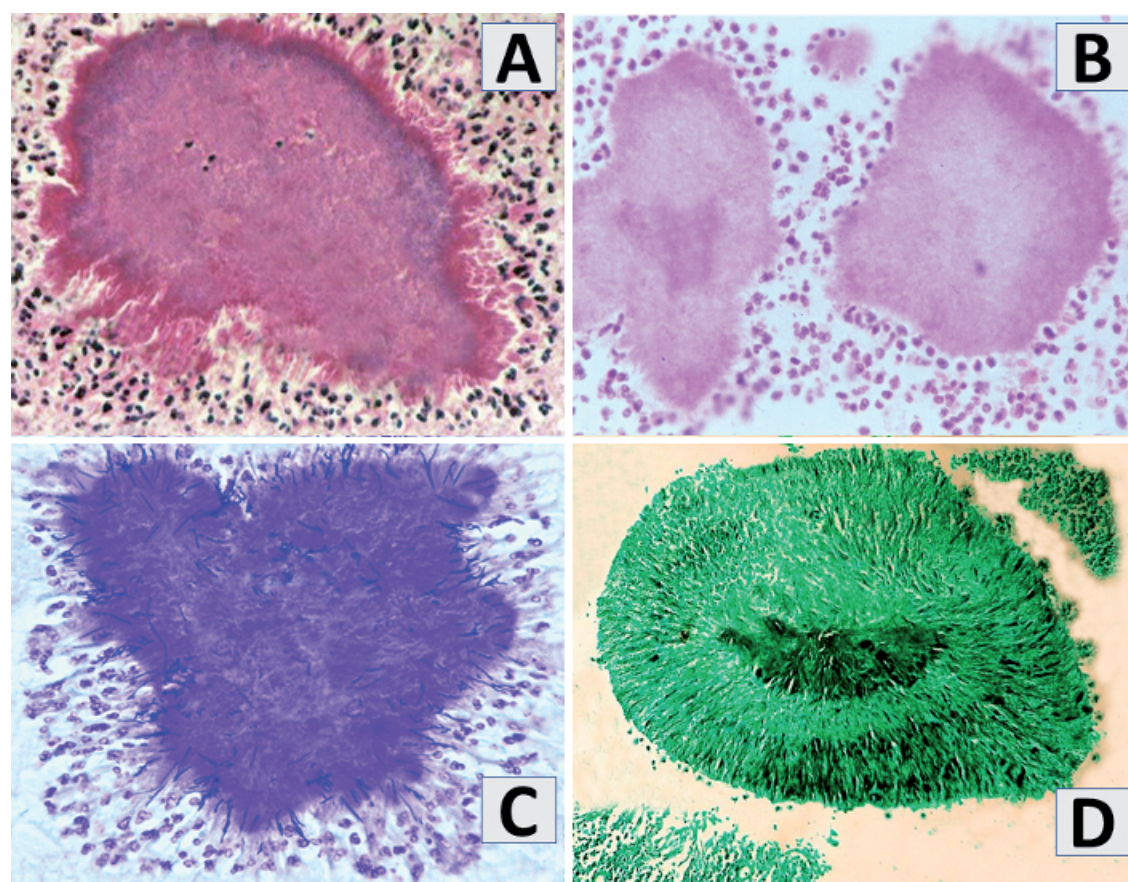

Figure 4. Cell blocks in pulmonary actinomycosis showing oval shaped sulfur granules with filiform bacilli mainly located at the periphery. A) H\&E stain, x200; B) PAS stain, x200; C) Giemsa stain, x200; D) Grocott-Gomori stain, x200. 
mycosis with endobronchial involvement: a case report and literature review. Tanaffos 2014;13:52-6.

14. Skehan N, Naeem M, Reddy RV. Endobronchial actinomycosis: successful treatment with oral antibiotics. BMJ Case Rep 2015;2015:bcr2015212754.

15. Higashi Y, Nakamura S, Ashizawa N, et al. Pulmonary actinomycosis mimicking pulmonary aspergilloma and a brief review of the literature. Intern Med 2017;56:449-53.

16. Gliga S, Devaux M, Gosset Woimant M, et al. Actinomyces graevenitzii pulmonary abscess mimicking tuberculosis in a healthy young man. Can Respir J 2014;21:e75-7.

17. Katsenos S, Galinos I, Styliara P, et al. Primary bronchopulmonary actinomycosis masquerading as lung cancer: Apropos of two cases and literature review. Case Rep Infect Dis 2015;2015:609637.

18. Bunkar ML, Gupta PR, Takhar R et al. Pulmonary actinomycosis masquerading as lung cancer: Case letter. Lung India 2016;33:460-2.

19. Boo YL, How KN, Pereira DS, et al. Pulmonary actinomycosis masquerading as lung cancer: A case report. Med J Malaysia 2017;72:246-7.

20. Bonnefond S, Catroux M, Melenotte $\mathrm{C}$ et al. Clinical features of actinomycosis: A retrospective, multicenter study of 28 cases of miscellaneous presentations. Medicine (Baltimore) 2016;95:e3923.

21. Han JY, Lee KN, Lee JK, et al. An overview of thoracic actinomycosis: CT features. Insights Imaging 2013;4:245-52.

22. Kim TS, Han J, Koh WJ et al. Thoracic actinomycosis: CT features with histopathologic correlation. AJR Am J Roentgenol 2006;186:225-31.

23. Valour F, Sénéchal A, Dupieux C et al. Actinomycosis: etiology, clinical features, diagnosis, treatment, and management. Infect Drug Resist 2014;7:183-97.

24. McHugh KE, Sturgis CD, Procop GW, Rhoads DD. The cytopathology of Actinomyces, Nocardia, and their mimickers. Diagn Cytopathol 2017;45:1105-15.

25. Kim SR, Jung LY, Oh IJ. et al. Pulmonary actinomycosis during the first decade of 21 st century: cases of 94 patients. BMC Infect Dis 2013;13:216.

26. Grzywa-Celińska A, Emeryk-Maksymiuk J, SzmyginMilanowska K, et al. Pulmonary actinomycosis - the great imitator. Ann Agric Environ Med 2017;25:211-2.

27. Zhang M, Zhang XY, Chen YB. Primary pulmonary actinomycosis: a retrospective analysis of 145 cases in mainland China. Int J Tuberc Lung Dis 2017;21:825-31.

28. Sun XF, Wang P, Liu HR, Shi JH. A retrospective study of pulmonary actinomycosis in a single institution in china. Chin Med J (Engl) 2015;128:1607-10.

29. Rosdina Z, Nurul Yaqeen ME, Hanafiah M, Nor Salmah B. Pulmonary actinomycosis masquerading as aspergilloma. Med J Malaysia 2017;72:147-9.

30. Rupani A, Amonkar G, Deshpande J. Pulmonary actinomycosismasquerading as tuberculosis. Indian J Pathol Microbiol 2009;52 438-9.

31. Kim YS, Suh JH, Kwak SM, et al. Foreign body-induced actinomycosis mimicking bronchogenic carcinoma. Korean J Intern Med 2002;17:207-10.
32. Imanishi S, Shinohara T, Naruse K, Ogushi F. Overlapping lung parenchymal and bronchial lesion and hilar lymphadenopathy in pulmonary actinomycosis mimicking lung cancer. BMJ Case Rep 2016;2016:bcr2016216308.

33. Olmez OF, Cubukcu E, Evrensel T, et al. Pulmonary actinomycosis mimicking metastasis from lung adenocarcinoma. Onkologie 2012;35:604-6.

34. Huang CW, Lee MA, Lu RH, et al. A case of pulmonary aspergilloma and actinomycosis. J Med Microbiol 2011;60: 543-6.

35. Lin L, Xue D, Lin TY, et al. Pulmonary aspergillosis, mucormycosis, and actinomycosis co-infection presenting as a cavitary lesion in a patient with diabetes. Chin Med J (Engl) 2019;132:2512-3.

36. Balis E, Kakavas S, Kompogiorgas S, et al. Presentation of pulmonary tuberculosis and actinomyces co-infection as a lung mass: a literature review and unique case report. Monaldi Arch Chest Dis 2019;89:1180.

37. Ghosh P, Gupta I, Kar M et al. Co-infection of Candida parapsilosis in a patient of pulmonary actinomycosis-A rare case report. J Clin Diagn Res 2017;11:DD01-2.

38. Oikonomidis P, Fousekis F, Kotsaftis P, et al. A case of pulmonary actinomycosis presented with endobronchial involvement. Respir Med Case Rep 2019;28:100930.

39. Supriya BG, Harisree S, Savio J, Ramachandran P. Actinomyces naeslundii causing pulmonary endobronchial actinomycosis - A case report. Indian J Pathol Microbiol 2019;62:326-8.

40. Seong GM, Hyun CL, Chang JW, Kim C. Unusual aetiology of lymphocyte-predominant exudative pleural effusion: primary mediastinal actinomycosis. Respirol Case Rep 2020;8:e0534.

41. Coodley EL, Yoshinaka R. Pleural effusion as the major manifestation of actinomycosis. Chest 1994;106:1615-7.

42. Wang L, Zhang H, Wu D et al. Pulmonary lesions associated with sputum culture-positive actinomycetes: report of one case. Ann Transl Med 2019;7:793.

43. Lazzari G, Vineis C, Cugini A. Cytologic diagnosis of primary pulmonary actinomycosis: report of two cases. Acta Cytol 1981;25:299-301.

44. Patel KB, Gupta G, Shah M, Patel P. Pulmonary actinomycosis in fine needle aspiration cytology. J Cytol 2009;26:94-6.

45. Crisafulli E, Bernardinello N, Alfieri V et al. A pulmonary infection by Actinomyces odontolyticus and Veillonella atypica in an immunocompetent patient with dental caries. Respirol Case Rep 2019;7:e00493.

46. Endo S, Mishima E, Takeuchi Y, et al. Periodontitis-associated septic pulmonary embolism caused by Actinomyces species identified by anaerobic culture of bronchoalveolar lavage fluid: a case report. BMC Infect Dis 2015;15:552.

47. Sharma S, Dey P, Poddar R. Pulmonary actinomycosis: a rare case diagnosed on bronchoalveolar lavage cytology. Cytopathology 2017;28:436-7.

48. Das DK. Actinomycosis in fine needle aspiration cytology. Cytopathology 1994;5:243-50.

49. de Montpréville VT, Nashashibi N, Dulmet EM. Actinomycosis and other bronchopulmonary infections with bacterial granules. Ann Diagn Pathol 1999;3:67-74. 